\title{
Evaluation of a direct method for the identification and antibiotic susceptibility assessment of microrganisms isolated from blood cultures by
} automatic systems

\author{
Sergio Frugoni', Giovanna Viola ${ }^{2}$, Eutilia Conte ${ }^{2}$, Daniela Consoli', Roberta Marchetti², \\ Chiara Silvia Vismara²
}

'ASP Istituti Milanesi Martinitt e Stelline e Pio Albergo Trivulzio, Servizio di Medicina di Laboratorio, via Trivulzio I5, 20146 Milano. ${ }^{2}$ Istituto Nazionale per lo Studio e la Cura dei Tumori, ${ }^{2}$ Medicina di Laboratorio via Venezian I, 20123 Milano.

Key word: Emocolture, survey direct

Valutazione di un metodo diretto per l'identificazione e l'accertamento della sensibilità agli antibiotici di microrganismi isolati da emocolture con sistemi automatici

\section{SUMMARY}

The purpose of blood cultures in the septic patient is to address a correct therapeutic approach. Identification and antibiotic susceptibility test carried out directly from the bottle may give important information in short time. The introduction of the automatic instrumentation has improved the discovering of pathogens in the blood, however the elapsing time between the positive detection and the microbiological report is still along. Is the evaluation of this study a fast, easy, cheap method to be applied to the routine, which could reduce the response time in the bacteraemia diagnosis. The automatic systems Vitek Senior (bioMérieux), andVitek 2 (bioMérieux) were used at Pio Albergo Trivulzio (Centrel) and at Istituto dei Tumori (Centre2) respectivetly. To remove blood cells, $7 \mathrm{ml}$. of the culture has been moved by vacuum sampling in a test tube and centrifuged for 10 minutes at $1000 \mathrm{rpm}$ the supernatant has been further centrifuged for 10 minutes at $3000 \mathrm{rpm} .0 .5 \mathrm{ml}$. of BHI has been added to the pellet o sediment. The concentration of bacterial suspension has been fit for the inoculation. At the same time has been prepared standard cultures in suitable culture media were carried out for comparison. In the centrol and centro2 have been isolated and identify respectively 63 and $31 \mathrm{Gram}$ negative, and, 32 and 40 gram positive microorganisms have been isolated and identify in the Centrel and Centre2 respectively. The identification Gram-negative and Gram positive microorganisms showed an agreement of $100 \%$ and $86.2 \%$ and $93.3 \%$ and $65.78 \%$ respectively between the direct and the standard method. For antibiotic susceptibility tests, 903 (Centrel) and 49I (Centre2) and 396 and 509 compounds were totally assessed in Gram negative and Gram positive bacteria respectively. The analysis has highlighted that: Centre I has reported $0.30 \%$ very major errors (GE), $0.92 \%$ major errors (EM), I.23\% minor errors (Em). Centre 2 showed $0.57 \%$ very major errors (GE), $0.09 \%$ major errors (EM), I.34\% minor errors (Em). The low percentage of GE of EM and Em obtained, the low cost and impact in the laboratory routine justify the use of a direct method with automatic instrumentations so that it allows to set up in short times an addressed antibiotic therapy.

\section{Received September 7, 2007}

Accepted November 5, 2007

\section{INTRODUZIONE}

Le batteriemie e le setticemie rappresentano ancor oggi uno dei principali problemi con conseguenze anche letali nel paziente ospedalizzato. Il ruolo dell'emocoltura nel paziente settico ha lo scopo fondamentale di indirizzare un corretto approccio terapeutico, al fine di impostare una terapia antibiotica mirata nel più breve tempo possibile.

Questa è una condizione importante soprattutto nell'ospite immunocompromesso poiché in tale paziente la terapia antibiotica empirica è resa più difficile dal fatto che l'eziologia delle infezioni appare spesso difficilmente ipotizzabile; inoltre le infezioni, anche se inizialmente localizzate, tendono velocemente a generalizzarsi e decorrono con andamento rapido.

I pazienti neoplastici in particolar modo sono a maggior rischio di infezione e la probabilità di sviluppare sepsi severe è da tre a cinque volte superiore rispetto ai pazienti non neoplastici (14).

\section{Corresponding author: Sergio Frugoni}

ASP I.M.M.eS.e P.A.T., - Servizio di Medicina di Laboratorio - Via Trivulzio I5 - 20146 Milano

Tel.: 024029544 - Fax: 024029549 - E-mail: sfrugoni@tiscalinet.it 
La chemioterapia, la radioterapia, l'alterazione della normale funzione dei linfociti o l'uso di corticosteroidi sono importanti fattori che compromettono il sistema immunitario e rendono il paziente neoplastico maggiormente esposto alle infezioni e alle relative complicazioni.

Anche l'anziano è notevolmente esposto al rischio di infezioni batteriche a causa di alterazioni delle capacità immunitarie $\mathrm{o}$ in seguito a malattie cronicodegenerative frequentemente presenti in questa fascia di età. In particolare l'incidenza delle sepsi severe nel paziente non neoplastico aumenta in modo significativo con l'aumentare dell'età (10) e diversi studi mostrano una chiara relazione tra incremento dell'età del paziente e mortalità associata a sepsi severe. Nei pazienti neoplastici invece l'età influisce in modo meno significativo sull'incidenza delle sepsi severe; questo può essere spiegato dal fatto che i pazienti più giovani sono sottoposti a terapie più aggressive rispetto a quelli più anziani.

Alla luce di questi dati risulta evidente come negli ospedali oncologici e nelle geriatrie, sia di fondamentale importanza la possibilità di eseguire una diagnosi microbiologica tempestiva che permetta di dare al clinico indicazioni precise rapidamente (3). Obiettivo principale dei microbiologi clinici dovrebbe essere infatti quello di caratterizzare il microrganismo e il pattern di sensibilità nel minor tempo possibile al fine di impostare quanto prima una terapia antibiotica mirata $(12,13)$. Non bisogna dimenticare che una diagnosi precoce, oltre ad abbassare il tasso di mortalità nei pazienti ospedalizzati, riduce notevolmente anche i costi legati alla terapia ed alla degenza in genere $(7,11)$.

L'introduzione negli anni novanta di strumentazioni automatiche per il rilevamento rapido di batteri e funghi nelle emocolture ha facilitato il riscontro di microrganismi patogeni nel sangue $(1,4,6)$. Tuttavia il tempo che intercorre tra il rilevamento automatico di positività e il referto microbiologico risulta ancora lungo.

Obiettivo del nostro lavoro è stato quello della messa a punto e della valutazione di un metodo rapido per la esecuzione delle emocolture, semplice, a basso costo, applicabile nella routine, che permetta di ridurre i tempi di risposta nella diagnosi delle batteriemie e fungemie.

\section{MATERIALI E METODI}

La valutazione è stata condotta presso i Laboratori di Analisi Chimico Cliniche e Microbiologiche dell'ASP Pio Albergo Trivulzio di Milano (Centro 1) e dell'Istituto Nazionale per lo Studio e la Cura dei Tumori di Milano (Centro 2). Ambedue le strutture di ricovero ospitano prevalentemente pazienti immunocompromessi in condizioni cliniche spesso gravi.

\section{Rilevazione delle emocolture positive}

La presenza di microrganismi nelle emocolture è stata rilevata in flaconi Bactec Plus Aerobic e Bactec Plus Anaerobic contenenti resine (Becton Dickinson) inoculati con protocolli standard di prelievo venoso e monitorati da Sistemi BACTEC (Becton Dickinson).

\section{Controllo di qualità}

Per il controllo dei test di sensibilità si sono utilizzati: E.coli ATCC 25922, P.aeruginosa ATCC 27853, S.aureus ATCC 29213, E.faecalis ATCC 29212, E.faecalis ATCC 51299.

Sono state approntate sospensioni batteriche dei ceppi con campioni di sangue di volontari sani.

Le sospensioni sono state inoculate in flaconi Bactec per aerobi e analizzate come normali campioni di emocoltura. I risultati sono stati confrontati con i valori CLSI presenti nel software di gestione aggiornati annualmente.

\section{Procedura analitica}

Dai flaconi positivi, in condizioni di sicurezza, sono stati prelevati $7 \mathrm{~mL}$ della coltura, trasferiti in provetta utilizzando sistemi di prelievo sottovuoto e centrifugati a $1000 \mathrm{rpm}$ per 10 minuti.

Il sovranatante separato è stato ulteriormente centrifugato a $3000 \mathrm{rpm}$ per 10 minuti; inoltre, con parte del sovranatante è stato allestito un vetrino per l'osservazione microscopica a fresco in campo scuro. Eliminato il sovranatante, con parte del precipitato è stato allestito un vetrino per la colorazione di Gram ed una reazione della citocromo ossidasi. Al precipitato sono stati poi aggiunti $0.5 \mathrm{~mL}$ di Brain Heart Infusion Broth (Becton Dickinson), la sospensione batterica ottenuta è stata portata alla concentrazione idonea per l'inoculo in strumentazioni automatiche. Con parte della sospensione, dove necessario, è stata allestita una prova della coagulasi libera.

Sono state contemporaneamente allestite colture standard in terreni idonei. Le procedure analitiche sono illustrate in figura I e II.

\section{Strumentazione automatica per identificazione ed antibiogramma}

Per l'identificazione dei microrganismi e per la determinazione della chemioantibiotico sensibilità sono state utilizzate strumentazioni (bioMérieux): Centro 1 - Vitek Senior, Centro 2 - Vitek 2.

La scelta delle card è conseguente alle caratteristiche del microrganismo evidenziato alla colorazione di Gram.

\section{Test aggiuntivi e di controllo}

Sono stati utilizzati test aggiuntivi o di controllo per migliorare o confermare i risultati delle strumentazioni automatiche.

Per migliorare l'identificazione dei microrganismi sono stati eseguiti, con metodologie standard, $i$ test aggiuntivi citocromo ossidasi e ricerca della coagulasi libera. 
La conferma dell'identificazione dei microrganismi è stata eseguita, dove necessario, con kit commerciali Api 20E (bioMérieux) per microrganismi gram negativi, Crystal GP (Becton Dikinson) per microrganismi gram positivi. Per la conferma della produzione di ß-lattamasi in Staphylococcus spp., sono stati utilizzati dischetti Cefinase (Becton Dikinson). La rilevazione delle $\beta$-lattamasi ad ampio spettro è stata eseguita con strip E-test (AB biodisck) secondo le metodologie standard.

\section{Definizioni}

Very major error (Falso sensibile) Major error (Falso resistente) Minor error sensibile metodo diretto resistente coltura in piastra resistente metodo diretto sensibile coltura in piastra sensibile o resistente metodo diretto intermedio coltura in piastra o viceversa

\section{RISULTATI}

Durante il periodo di valutazione del metodo, non sono state osservate batteriemie ad eziologia polimicrobica. L'osservazione a fresco, la colorazione di Gram e successivamente la coltura in piastra ci hanno permesso di escludere la possibile presenza di più di un microrganismo.

Nel Centro1 sono stati isolati 95 microrganismi; 63 Gram negativi e 32 Gram positivi. Si può evidenziare che su 95 identificazioni eseguite con metodo diretto utilizzando lo strumento Vitek Senior, 93 (97.9\%) concordano con il risultato ottenuto dalla coltura in piastra. 71 sono stati i microrganismi isolati nel Centro2; 31 Gram negativi, 40 Gram positivi. Con Vitek2 sono stati identificati correttamente $58(81.7 \%)$ microrganismi. Nelle tabelle 1 e 2 sono riportati i microrganismi isolati ed identificati con il metodo diretto e confrontati con la coltura in piastra di entrambi i cen-
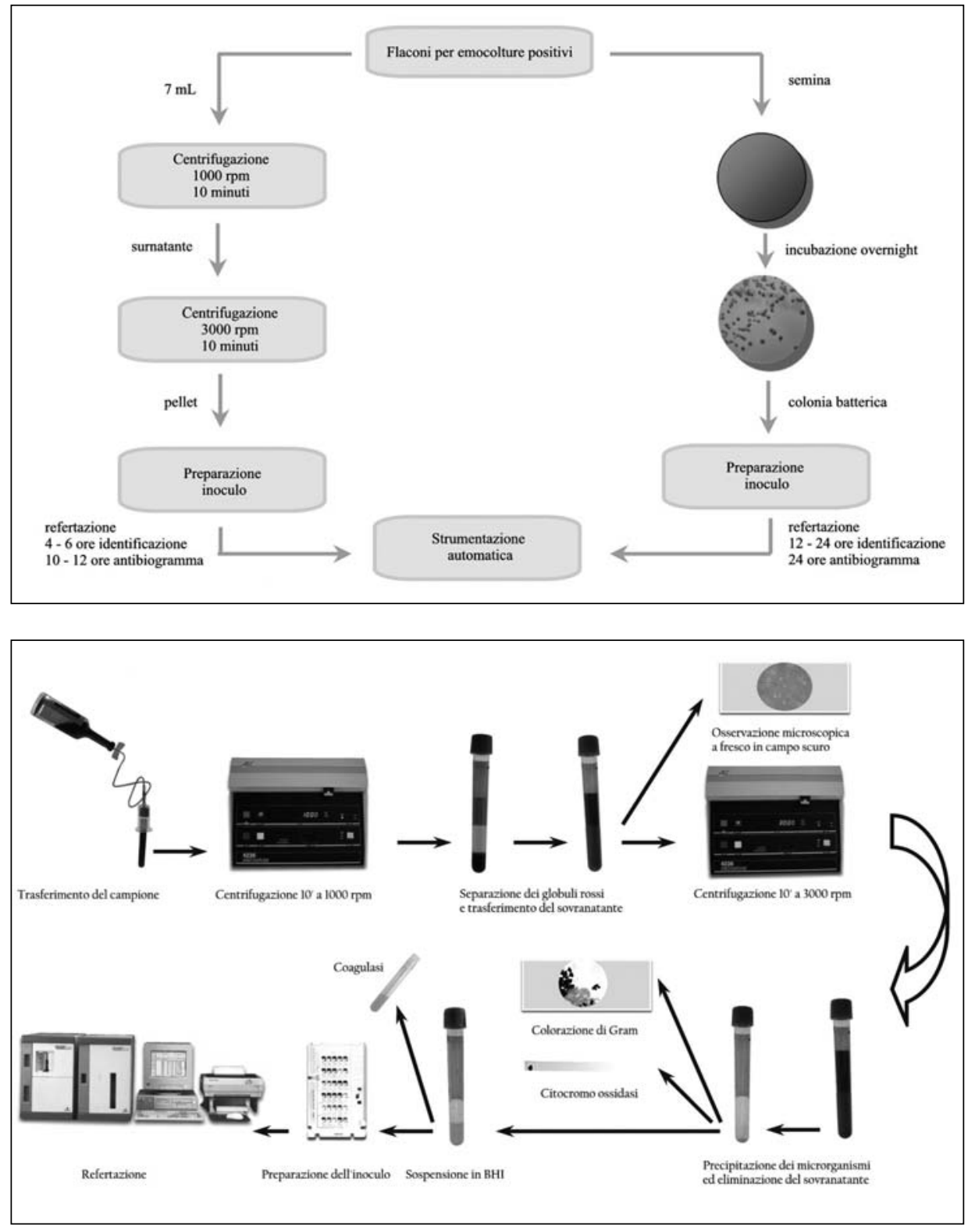

Figura I

Diagramma di flusso delle procedure eseguite

Figura II

Metodo diretto - procedura analitica 
tri. Gli errori di identificazione o l'impossibilità di identificare un ceppo riguardano in entrambi gli strumenti quasi esclusivamente i batteri Gram positivi, ed in particolare Stafilococchi. Su 160 dei 166 ceppi isolati è stato eseguito l'antibiogramma. I microrganismi sui quali non è stata eseguita la valutazione della sensibilità con strumentazione automatica sono Streptococcus pneumoniae, Bacteroides capillosus, Micrococcus luteus, Brevundimonas diminuta e Candida albicans. Questi ceppi sono comunque stati identificati correttamente con metodo diretto.

Le tabelle 3 e 4 mostrano i risultati del confronto dei test di sensibilità agli antibiotici ottenuti con metodo diretto e metodo standard (coltura in piastra) nei diversi ceppi batterici isolati. I risultati sono espressi in percentuale di errore rispetto al totale di combinazioni di antibiotici testati per ogni ceppo batterico. Con Vitek Senior, 20 (21.7\%) dei 92 ceppi isolati mostrano una completa concordanza di risultati per tutte le combinazioni antibiotico/microrganismo testate: non si sono mai verificati "very major error", "major error" e "minor error". Con Vitek2 i ceppi che non mostrano discordanze nei test di sensibilità sono 27 (39.7\%). Il confronto tra metodo diretto e coltura in piastra dei test di sensibilità riferiti ai singoli antibiotici sono rappresentati nelle tabelle 5 e 6 . I risultati, sono espressi come percentuale di errore sul totale di combinazioni antibiotico/microrganismo testate per classe di chemioterapico. Con Vitek Senior su 62 ceppi di Gram negativi isolati, sono state testate 903 combinazioni antibiotico/microrganismo. La percentuale di "very major error", "major error" e "minor error" è stata rispettivamente di $0.2 \%$, $0.9 \%, 1.1 \%$.

Con Vitek2 su 30 ceppi di Gram negativi isolati, sono state testate 491 combinazioni antibiotico/microrganismo. La percentuale di "very major error", "major error" e "minor error" è stata rispettivamente di $0.8 \%, 0 \%, 1.6 \%$.

Per i microrganismi Gram negativi, la percentuale complessiva di errore è pari al $2.2 \%$ per Vitek Senior e a $2.4 \%$ per Vitek2. I "very major error" in entrambi gli strumenti riguardano i risultati dei test di sensibilità di penicilline e cefalosporine di terza e quarta generazione; in particolare Piperacillina, Piperacillina/tazobactam, Ceftazidime, Cefepime per Vitek2 e Cefotaxime per Vitek Senior.

Per quanto riguarda i Gram positivi sono stati testati 30 ceppi e 396 combinazioni antibiotico/microrganismo con Vitek Senior e 38 ceppi e 509 combinazioni antibiotico/microrganismo con Vitek2. La percentuale di "very major error", "major error" e "minor error" sul totale degli antibiotici testati è stata rispettivamente di $0.5 \%, 1.0 \%, 1.5 \%$ per Vitek Senior e $0.4 \%, 0.2 \%, 1.2 \%$ per Vitek 2 .
Tabella I. Vitek Senior: identificazione di 93 isolati da emocolture positive mediante metodo diretto e metodo standard

\begin{tabular}{lcccc}
\hline Microrganismi & $\begin{array}{c}\text { Identificazione } \\
\text { corretta }\end{array}$ & $\begin{array}{c}\text { Identificazione } \\
\text { non corretta }\end{array}$ & Totale \\
\hline Acinetobacter baumannii & $\mathrm{I}$ & 0 & $\mathrm{I}$ \\
\hline Escherichia coli & 29 & 0 & 29 \\
\hline Klebsiella pneumoniae & 10 & 0 & 10 \\
\hline Pseudomonas aeruginosa & 8 & 0 & 8 \\
\hline Proteus mirabilis & 14 & 0 & 14 \\
\hline Bacteroides capillosus & $\mathrm{I}$ & 0 & $\mathrm{I}$ \\
\hline Enterococcus faecalis & 6 & 0 & 6 \\
\hline Staphylococcus aureus & 10 & 0 & 10 \\
\hline Staphylococcus epidermidis & 8 & 1 & 9 \\
\hline Staphylococcus hominis & 2 & 0 & 2 \\
\hline Streptococcus pneumoniae & $\mathrm{I}$ & 0 & $\mathrm{I}$ \\
\hline Staphylococcus sciuri & $\mathrm{I}$ & 0 & $\mathrm{I}$ \\
\hline Staphylococcus simulans & $\mathrm{I}$ & 0 & $\mathrm{I}$ \\
\hline Staphylococcus warneri & 0 & 1 & $\mathrm{I}$ \\
\hline Candida albicans & $\mathrm{I}$ & 0 & $\mathrm{I}$ \\
\hline Totale (\%) & $93(97.9)$ & $2(2.1)$ & 95 \\
\hline
\end{tabular}

Tabella II. Vitek 2: identificazione di 58 isolati da emocolture positive mediante metodo diretto e metodo standard

\begin{tabular}{lccc} 
Microrganismi & $\begin{array}{c}\text { Identificazione } \\
\text { corretta }\end{array}$ & $\begin{array}{c}\text { Identificazione } \\
\text { non corretta }\end{array}$ & Totale \\
\hline Brevundimonas diminuita & I & 0 & I \\
\hline Citrobacter diversus & I & 0 & I \\
\hline Enterobacter cloacae & 2 & 0 & 2 \\
\hline Escherichia coli & 8 & 0 & 8 \\
\hline Klebsiella oxytoca & I & 0 & I \\
\hline Pseudomonas aeruginosa & 9 & 2 & II \\
\hline R.pickettii & 1 & 0 & I \\
\hline Stenotrophomonas maltopilia & 3 & 0 & 3 \\
\hline Serratia marcescens & 3 & 0 & 3 \\
\hline Enterococcus faecalis & 2 & 0 & 2 \\
\hline Enterococcus faecium & 0 & 1 & I \\
\hline Micrococcus luteus & 1 & 0 & I \\
\hline Streptococcus agalactiae & 2 & 0 & 2 \\
\hline Staphylococcus aureus & 4 & 2 & 6 \\
\hline Staphylococcus epidermidis & 12 & 4 & 16 \\
\hline Staphylococcus haemolyticus & 3 & 2 & 5 \\
\hline Staphylococcus hominis & 4 & 2 & 6 \\
\hline Streptococcus pneumoniae & 1 & 0 & I \\
\hline Totale (\%) & $58(81.7)$ & $13(18.3)$ & 71 \\
\hline
\end{tabular}

Per i microrganismi Gram positivi, la percentuale complessiva di errore è pari al $3 \%$ per Vitek Senior e a $1.8 \%$ per Vitek2. I "very major error" in entrambi gli strumenti riguardano i risultati dei test di sensibilità di Eritromicina per entrambi gli strumenti, Rifampicina per Vitek Senior, Oxacillina per Vitek2.

Dall'analisi complessiva dei risultati ottenuti dalle combinazioni antibiotico/microrganismo di tutti i microrganismi testati si evidenzia: Vitek Senior - "very major error" $0.30 \%$, "major error" $0.92 \%$, "minor error" $1.23 \%$.

Vitek 2 - "very major error" $0.57 \%$, "major error" $0.09 \%$, "minor error" $1.34 \%$.

Nei controlli di qualità eseguiti con metodo diret- 
Tabella 3. Vitek Senior. Sensibilità agli antibiotici per specie isolata: confronto tra metodo diretto e ceppi da coltura

\begin{tabular}{|c|c|c|c|c|}
\hline Microrganismi & $\mathbf{N}^{\circ}$ ceppi testati & \% very major errors & \% major error & $\%$ minor errors \\
\hline \multicolumn{5}{|l|}{ Gram negativi } \\
\hline Acinetobacter baumannii & $\mathrm{I}$ & 0 & 0 & 0 \\
\hline Escherichia coli & 29 & 0 & 0 & 0.9 \\
\hline Proteus mirabilis & 14 & 1.0 & 1.0 & 3.0 \\
\hline Klebsiella pneumoniae & 10 & 0 & 0 & 0 \\
\hline Pseudomonas aeruginosa & 8 & 0 & 5.3 & 0 \\
\hline Totale & 62 & 0.2 & 0.9 & I.I \\
\hline \multicolumn{5}{|l|}{ Gram positivi } \\
\hline Staphylococcus aureus & 10 & 0.7 & 0 & 2.2 \\
\hline Staphylococcus epidermidis & 9 & 0.8 & 0 & 2.4 \\
\hline Staphylococcus hominis & 2 & 0 & 14.8 & 0 \\
\hline Staphylococcus sciuri & $\mathrm{I}$ & 0 & 0 & 0 \\
\hline Staphylococcus simulans & I & 0 & 0 & 0 \\
\hline Staphylococcus warneri & $\mathrm{I}$ & 0 & 0 & 0 \\
\hline Enterococcus faecalis & 6 & 0 & 0 & 0 \\
\hline Totale & 30 & 0.5 & 1.0 & 1.5 \\
\hline
\end{tabular}

Tabella 4. Vitek 2. Sensibilità agli antibiotici per specie isolata: confronto tra metodo diretto e ceppi da coltura.

\begin{tabular}{|c|c|c|c|c|}
\hline Microrganismi & $\mathbf{N}^{\circ}$ ceppi testati & \% very major errors & $\%$ major error & $\%$ minor errors \\
\hline \multicolumn{5}{|l|}{ Gram negativi } \\
\hline Citrobacter diversus & $\mathrm{I}$ & 0 & 0 & 5.0 \\
\hline Enterobacter cloacae & 2 & 0 & 0 & 2.6 \\
\hline Escherichia coli & 8 & 0 & 0 & 0 \\
\hline Klebsiella oxytoca & $\mathrm{I}$ & 0 & 0 & 0 \\
\hline Pseudomonas aeruginosa & 11 & 2.0 & 0 & 3.1 \\
\hline Stenotrophomonas maltophilia & 3 & 0 & 0 & 0 \\
\hline Ralstonia picketti & $\mathrm{I}$ & 0 & 0 & 0 \\
\hline Serratia marcescens & 3 & 0 & 0 & 0 \\
\hline Totale & 30 & 0.8 & 0 & 1.6 \\
\hline \multicolumn{5}{|l|}{ Gram positivi } \\
\hline Staphylococcus aureus & 6 & 0 & 0 & 0 \\
\hline Staphylococcus epidermidis & 16 & 0 & 0 & 0.5 \\
\hline Staphylococcus haemolyticus & 5 & 1.5 & 0 & 0 \\
\hline Staphylococcus hominis & 6 & 1.1 & 1.1 & 3.4 \\
\hline Enterococcus faecalis & 2 & 0 & 0 & 0 \\
\hline Enterococcus faecium & 1 & 0 & 0 & 0 \\
\hline Streptococcus agalactiae & 2 & 0 & 0 & 0 \\
\hline Totale & 38 & 0.4 & 0.2 & 1.2 \\
\hline
\end{tabular}

to, non sono state osservate discrepanze tra i valori ottenuti e quelli tabulati CLSI.

La produzione di ß-lattamasi in Staphylococcus spp., così come la produzione di ß-lattamasi ad ampio spettro in microrganismi Gram negativi, è stata correttamente rilevata dalla strumentazione automatica in tutti i campioni esaminati.

\section{DISCUSSIONE}

L'esigenza di ottenere quanto prima il risultato relativo ad una emocoltura positiva dovrebbe essere uno degli obiettivi prioritari in microbiologia, per incidere in maniera considerevole sulla scelta del farmaco e ridurre se possibile la mortalità. Il nostro lavoro ha voluto valutare la riduzione del tempo di refertazione utilizzando metodi automatizzati normalmente usati in routine rendendoli però più efficienti mediante l'allestimento di identificazione ed antibiogramma direttamente da flacone positivo anziché da piastra.
Una lettura attenta e approfondita dei vetrini preparati a fresco e con colorazione di Gram permette, non solo di individuare batteriemie a eziologia polimicrobica, ma di utilizzare card specifiche ed appropriate per ciascun "gruppo" di microrganismi, con evidente riduzione dei costi.

La metodologia da noi seguita, non può essere applicata a campioni con presenza di flora batterica mista.

I dati relativi alla nostra esperienza mostrano un'elevata percentuale complessiva di identificazioni corrette (91\%) ottenute con il metodo diretto. In particolare 1'82.2\% di tutti i ceppi Gram positivi e il $97.8 \%$ di tutti i ceppi Gram negativi sono stati identificati correttamente.

Per quanto riguarda microrganismi Gram negativi, l'identificazione diretta mostra una completa attendibilità, in linea con i dati della letteratura $(2$, $5,9)$. Per i Gram positivi i nostri dati si discostano dai risultati riportati in studi precedenti e 
Tabella 5. Bacilli Gram negativi. Sensibilità agli antibiotici: confronto tra metodo diretto e ceppi isolati in coltura.

(*) antibiotico non testato

\begin{tabular}{|c|c|c|c|c|c|c|c|c|}
\hline \multirow[b]{2}{*}{ Antibiotico } & \multicolumn{4}{|c|}{ VitekSenior } & \multicolumn{4}{|c|}{ Vitek 2} \\
\hline & $\begin{array}{l}\text { Numero } \\
\text { ceppi } \\
\text { testati }\end{array}$ & $\begin{array}{c}\text { Numero } \\
\text { very major } \\
\text { error }\end{array}$ & $\begin{array}{c}\text { Numero } \\
\text { major } \\
\text { errors }\end{array}$ & $\begin{array}{l}\text { Numero } \\
\text { minor } \\
\text { errors }\end{array}$ & $\begin{array}{l}\text { Numero } \\
\text { ceppi } \\
\text { testati }\end{array}$ & $\begin{array}{c}\text { Numero } \\
\text { very major } \\
\text { errors }\end{array}$ & $\begin{array}{c}\text { Numero } \\
\text { major } \\
\text { error }\end{array}$ & $\begin{array}{l}\text { Numero } \\
\text { minor } \\
\text { errors }\end{array}$ \\
\hline Amikacina & 62 & 0 & 0 & 0 & 18 & 0 & 0 & 1 \\
\hline Amoxicillina/Ac.Clav. & $\begin{array}{ll}\text { v. } 62 \\
\end{array}$ & 0 & 0 & 2 & 17 & 0 & 0 & 0 \\
\hline Ampicillina & $-(*)$ & - & - & - & 27 & 0 & 0 & 0 \\
\hline Ampicillina/Sulbac. & - & - & - & - & 12 & 0 & 0 & 0 \\
\hline Aztreonam & 58 & 0 & 2 & 2 & - & - & - & - \\
\hline Cefalotina & 60 & 0 & 0 & 0 & 17 & 0 & 0 & 0 \\
\hline Cefazolina & - & - & - & - & II & 0 & 0 & 0 \\
\hline Cefepime & - & - & - & - & 14 & 1 & 0 & 0 \\
\hline Cefixime & - & - & - & - & 11 & 0 & 0 & 0 \\
\hline Cefotaxime & 58 & 2 & 0 & 0 & 26 & 0 & 0 & $\mathrm{I}$ \\
\hline Cefoxitina & - & - & - & - & 22 & 0 & 0 & $\mathrm{I}$ \\
\hline Cefpodoxime & - & - & - & - & 9 & 0 & 0 & 0 \\
\hline Ceftazidime & 60 & 0 & 0 & 0 & 28 & $\mathrm{I}$ & 0 & $\mathrm{I}$ \\
\hline Ceftriaxone & 53 & 0 & 0 & 0 & - & - & - & - \\
\hline Ciprofloxacina & 62 & 0 & 2 & 0 & 29 & 0 & 0 & 0 \\
\hline Gentamicina & 62 & 0 & 2 & 0 & 29 & 0 & 0 & 0 \\
\hline Imipenem & 62 & 0 & 0 & 0 & 22 & 0 & 0 & 2 \\
\hline Levofloxacina & 60 & 0 & 2 & 0 & 12 & 0 & 0 & 0 \\
\hline Meropenem & - & - & - & - & 19 & 0 & 0 & 0 \\
\hline Mezlocillina & - & - & - & - & $\mathrm{II}$ & 0 & 0 & 0 \\
\hline Netilmicina & - & - & - & - & 9 & 0 & 0 & 0 \\
\hline Ofloxacina & - & - & - & - & 16 & 0 & 0 & $\mathrm{I}$ \\
\hline Piperacillina & 62 & 0 & 0 & 2 & 16 & $\mathrm{I}$ & 0 & 0 \\
\hline Piperacillina/Tazobac & - & - & - & - & 29 & $\mathrm{I}$ & 0 & 0 \\
\hline Tetraciclina & - & - & - & - & 12 & 0 & 0 & $\mathrm{I}$ \\
\hline Ticarcillina & - & - & - & - & 8 & 0 & 0 & 0 \\
\hline Ticarcillina/Ac.Clav. & 60 & 0 & 0 & 2 & 9 & 0 & 0 & 0 \\
\hline Tobramicina & 60 & 0 & 0 & 2 & 29 & 0 & 0 & 0 \\
\hline Trimetoprim/Sulfam. & 62 & 0 & 0 & 0 & 29 & 0 & 0 & 0 \\
\hline Totale (\%) & 903 & $2(0.2)$ & $8(0.9)$ & $10(1.1)$ & 491 & $4(0.8)$ & $0(0)$ & $8(1.6)$ \\
\hline
\end{tabular}

Tabella 6. Bacilli Gram positivi. Sensibilità agli antibiotici: confronto tra metodo diretto e ceppi isolati in coltura. (*) antibiotico non testato.

\begin{tabular}{|c|c|c|c|c|c|c|c|c|}
\hline \multirow[b]{2}{*}{ Antibiotico } & \multicolumn{4}{|c|}{$\begin{array}{l}\text { VitekSenior } \\
\end{array}$} & \multicolumn{4}{|c|}{ Vitek 2} \\
\hline & $\begin{array}{l}\text { Numero } \\
\text { ceppi } \\
\text { testati }\end{array}$ & $\begin{array}{c}\text { Numero } \\
\text { very major } \\
\text { error }\end{array}$ & $\begin{array}{c}\text { Numero } \\
\text { major } \\
\text { errors }\end{array}$ & $\begin{array}{c}\text { Numero } \\
\text { minor } \\
\text { errors }\end{array}$ & $\begin{array}{c}\text { Numero } \\
\text { ceppi } \\
\text { testati }\end{array}$ & $\begin{array}{c}\text { Numero } \\
\text { very major } \\
\text { errors }\end{array}$ & $\begin{array}{c}\text { Numero } \\
\text { major } \\
\text { error }\end{array}$ & $\begin{array}{c}\text { Numero } \\
\text { minor } \\
\text { errors }\end{array}$ \\
\hline Ampicillina & $-(*)$ & - & - & - & 5 & 0 & 0 & 0 \\
\hline Ciprofloxacina & - & - & - & - & 3 & 0 & 0 & 0 \\
\hline Clindamicina & 24 & 0 & 0 & 0 & 36 & 0 & 0 & 0 \\
\hline Eritromicina & 23 & I & 0 & 0 & 39 & $\mathrm{I}$ & 0 & 0 \\
\hline Gentamicina & 23 & 0 & I & I & 32 & 0 & 0 & 2 \\
\hline Gentamicina high dos & sse 6 & 0 & 0 & 0 & 3 & 0 & 0 & 0 \\
\hline Imipenem & 6 & 0 & 0 & 0 & 4 & 0 & 0 & 0 \\
\hline Kanamicina & - & - & - & - & 8 & 0 & 0 & 0 \\
\hline Kanamicina high dose & & & & & 3 & 0 & 0 & 0 \\
\hline Levofloxacina & 27 & 0 & 1 & 1 & 5 & 0 & 0 & 0 \\
\hline Linezolid & 30 & 0 & 0 & 0 & - & - & - & - \\
\hline Minociclina & - & - & - & - & 34 & 0 & 0 & 0 \\
\hline Moxifloxacina & 30 & 0 & $\mathrm{I}$ & 2 & - & - & - & - \\
\hline Ofloxacina & - & - & - & - & 39 & 0 & 0 & 2 \\
\hline Oxacillina & 24 & 0 & 0 & 0 & 33 & I & 0 & 0 \\
\hline Penicillina G & 30 & 0 & 0 & 0 & 38 & 0 & 0 & 0 \\
\hline Quinupristin/Dalfop. & 30 & 0 & 0 & 0 & 5 & 0 & 0 & 0 \\
\hline Rifampicina & 23 & 1 & I & I & 34 & 0 & 0 & 0 \\
\hline Streptomicina & 6 & 0 & 0 & 0 & 3 & 0 & 0 & 0 \\
\hline Teicoplanina & 30 & 0 & 0 & 0 & 39 & 0 & 0 & I \\
\hline Tetraciclina & 30 & 0 & 0 & $\mathrm{I}$ & 37 & 0 & 0 & 0 \\
\hline Tobramicina & & & & & 33 & 0 & I & I \\
\hline Trimetoprim/Sulfam. & 24 & 0 & 0 & 0 & 37 & 0 & 0 & 0 \\
\hline Vancomicina & 30 & 0 & 0 & 0 & 39 & 0 & 0 & 0 \\
\hline Totale (\%) & 396 & $2(0.5)$ & $4(1.0)$ & $6(1.5)$ & 509 & $2(0.4)$ & $I(0.2)$ & $6(1.2)$ \\
\hline
\end{tabular}


mostrano una buona concordanza tra metodo diretto e coltura in piastra, anche se inferiore a quella dei Gram negativi.

Data questa elevata correlazione tra i due metodi e la notevole riduzione dei tempi di identificazione (entro 6 ore dalla positività della coltura per il metodo diretto rispetto alle oltre 24 ore del metodo in piastra), risulta di grande utilità inviare al medico curante un referto preliminare riportante l'esito dell'esame microscopico e l'identificazione data dalla strumentazione automatica.

Seppur con valori differenti, i dati ottenuti con le due diverse strumentazioni mostrano, in linea con la letteratura internazionale, una bassa percentuale di "very major error", "major error" e "minor error". Gli antibiotici che mostrano la più alta percentuale di "very major error" per i microrganismi Gram negativi sono i ß-lattamici ad ampio spettro ed in particolare le stesse molecole che, secondo i dati della letteratura (8), presentano una minor accuratezza nel risultato ottenuto con metodi automatici. I tempi di risposta ridotti, il basso costo e lo scarso impatto che il metodo presenta nella routine di laboratorio oltre che l'ottima performance, giustificano l'utilizzo della metodica da noi valutata, poiché permette di impostare una corretta e mirata terapia in tempi brevi.

La metodologia qui riportata è stata inserita nei manuali di microbiologia di entrambi i Centri.

\section{BIBLIOGRAFIA}

1. Berhin C, Glupczynski Y, De Gheldre Y. Evalutation of the Vitek 2 system for rapid direct identification and susceptibility testing from positive blood culture. Clinical Microbiol. And Inf. 2004.

2. Brins MJ, Bloembergen P, Ruijs GJHM, Wolfhagen MJHM. Identification and Susceptibility testing of enterobacteriaceae and Pseudomonas aeruginosa by direct inoculation from positive bactec blood culture bottles into Vitek 2. J Clinical Microbiol. 2004; 42: 711.

3. Conte E, Vismara C, Corengia V, Carati MR, Viola G. Valutazione di un metodo diretto per l'esecuzione di identificazione ed antibiogramma da emocolture con sistema VITEK 2. Poster M030, XXXII Congresso Nazionale Associazione Microbiologi Clinici Italiani, Firenze 2003.

4. De Cueto M, Ceballos E, Martinez-ML, Perea EJ, Pasqual A. Use of positive blood culture for direct identification and susceptibility testing with the Vitek 2 system. J Clin Microbiol 2004; 42: 3734-8.

5. Fontanals D, Salceda F, Hernandez J, Sanfeliu I, Torra M. Evaluation of Wider system for direct identification and antimicrobial susceptibility testing of Gramnegative bacilli from positive blood culture bottles. Eur J Clin Microbiol Infect Dis 2002; 21: 693-5.

6. Funke G, Funke-Kissling P. Use of the BD PHOENIX automated microbiology system for direct identification and susceptibility testing of Gram-negative rods from positive blood cultures in thre-phase trial. J Clin Microbiol 2004; 42: 1466-70.
7. Hansen DS, Jensen AG, Norskov-Lauritsen N, Skov R, Bruun B. Direct identification and susceptibility testing of enteric bacilli from positive blood cultures using VITEK (GNI+/GNS-GA). Clin Microbiol Infect 2002; 8: 38-44.

8. Juretschko S, LaBombardi VJ, Lerner SA. Schreckenberger Accuracy of B-Lactam Susceptibility Testing Results J Clin Microbiol 2007.

9. Ling TKW, Liu ZK, Cheng FB. Evaluation of the VITEK 2 system for rapid direct identification and susceptibility testing of Gram-negative bacilli from positive blood cultures. J Clin Microbiol 2003; 41: 4705-7.

10. Martin GS, Mannino DM, Moss M. The effect of age on the development and outcome of adult sepsis. Crit Care Med 2006; 34: 15-21.

11. Putman LR, Hovard WJ, Pfaller MA, Koontz FP, Jones RN. Accuracy of the VITEK system for antimicrobial susceptibility testing of Enterobatteriaceae bliidstream infection isolates: use of "direct" inoculation from Bactec 9240 blood culture bottles. Diagn Microbiol Infect Dis 1997; 28: 101-4.

12. Waites KB, Brookings ES, Moser SA, Zimmer BL. Direct bacterial identification from positive BacT/Alert blood cultures using MicroScan overnight and rapid panels. Diagn Microbiol Infect Dis. 1998; 32: 21-6.

13. Waites KB, Brookings ES, Moser SA, Zimmer BL. Williams MD, Braun LA, Cooper LM, et al. Hospitalized cancer patiens with severe sepsis: analysis of incidence, mortality, and associated cost of care. Critical Care 2004; 8: 291-8. 